\title{
Hypersensitivity Pneumonitis: A Woman With Chronic Dyspnea
}

\author{
Naketa Chand ${ }^{\mathrm{a}}$, Krishna Gollamudi ${ }^{\mathrm{b}}$, Zuha'a Khan ${ }^{\mathrm{c}}$, \\ Roohab Shaikh ${ }^{\mathrm{d}, \mathrm{e}}$
}

\begin{abstract}
Bird fancier's lung is a type of hypersensitivity pneumonitis, commonly seen after being exposed to avian antigens. Herein, we describe a 37-year-old female who was diagnosed with bird fancier's lung after purchasing three cockatiels. She presented with dyspnea, fever, fatigue and cough and the diagnosis was confirmed by a series of tests including pulmonary function tests, chest X-ray and bronchoalveolar lavage indicating lymphocytosis and increase in mast cells. Our case demonstrated the entire course of the disease along with various treatment options.
\end{abstract}

Keywords: Hypersensitivity; Pneumonitis; Bird fancier's lung

\section{Introduction}

Bird fancier's lung (BFL) is one of the most common causes of hypersensitivity pneumonitis (HP), and accounts for $66-68 \%$ of all HP. BFL most commonly results from exposure to aerosolized avian proteins, specifically, feathers and droppings. Prolonged exposure triggers an immune-complex mediated hypersensitivity reaction which leads to the development of clinical manifestations including dyspnea, cough, fever, and fatigue. Although there is no one specific test for a definitive diagnosis, it is commonly diagnosed through clinical presentation, pulmonary function tests demonstrating a restrictive pattern, chest X-ray, increased lymphocytes on bronchoalveolar lavage and lung biopsies, if needed. BFL is commonly treated

Manuscript submitted September 6, 2017, accepted October 12, 2017

${ }^{a}$ Xavier University School of Medicine, 1000 Woodbury Road, Suite 109, Woodbury, NY 11797, USA

bAvalon University School of Medicine, Churchill Hubbard Rd, Youngstown, $\mathrm{OH} 44505$, USA

${ }^{\mathrm{c} C a r i b b e a n ~ M e d i c a l ~ U n i v e r s i t y, ~} 5600$ N River Road Suite 800, Des Plaines, IL 60018, USA

${ }^{\mathrm{d} W i n d s o r}$ University School of Medicine, 6212 Monee Manhattan Rd, Monee, IL 60449, USA

'Corresponding Author: Roohab Shaikh, Windsor University School of Medicine, 6212 Monee Manhattan Rd, Monee, IL 60449, USA.

Email: roohab.shaikh@gmail.com

doi: https://doi.org/10.14740/jmc2928e by identifying and decreasing exposure to the precipitating factor.

\section{Case Report}

A 37-year-old female was seen in the clinic complaining of progressive shortness of breath on exertion for 8 months. The shortness of breath improved when she went on a work-related trip but returned within a few hours upon her return home. She had a history of cigarette use of 11 pack-years. She denied any unintentional weight loss and changes in appetite. Physical examination revealed no digital clubbing and chest auscultation revealed late-inspiratory crackles at the lung bases posteriorly. Pulmonary function tests (PFTs) showed a restrictive and obstructive pattern and high resolution computed tomography (HRCT) of the chest showed diffuse micronodules and ground-glass attenuation (Fig. 1). Specific IgG antibodies (precipitins) were negative. Flexible bronchoscopy with bronchoalveolar lavage (BAL) were performed which illustrated $64 \%$ lymphocytosis with $1.8 \%$ mast cells (Table 1 ). Upon further questioning patient stated she purchased three cockatiel birds within the last year. The overall clinical presentation was

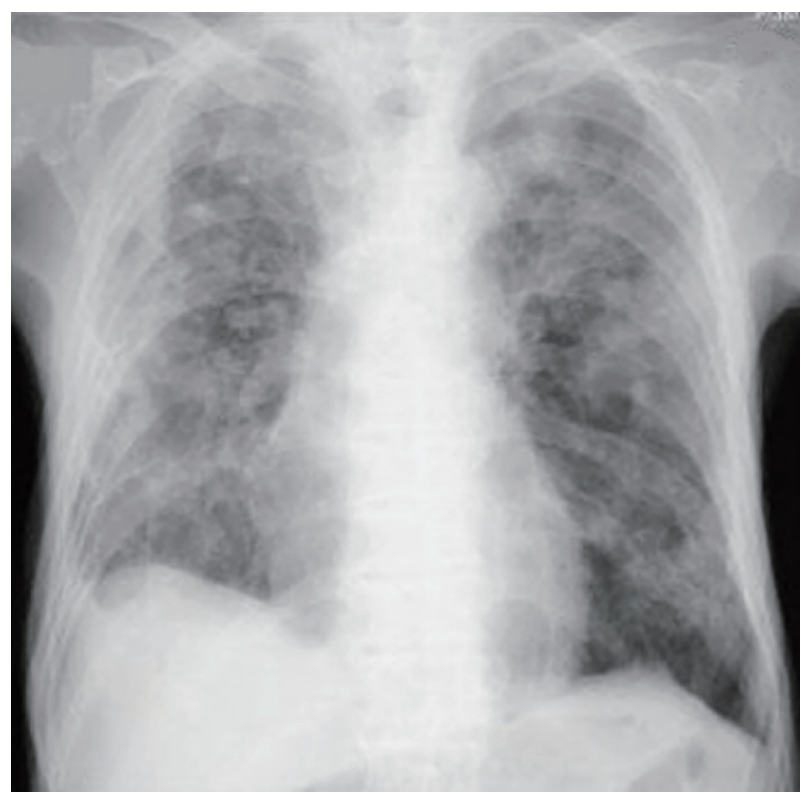

Figure 1. Chest X-ray showing multiple ground-glass opacities. 
Table 1. Lab Results

\begin{tabular}{|c|c|}
\hline Laboratory examinations & Results \\
\hline \multicolumn{2}{|l|}{$\mathrm{CBC}$} \\
\hline $\mathrm{RBC}$ count & 5.31 \\
\hline Hemoglobin & $14.7 \mathrm{~g} / \mathrm{dL}$ \\
\hline Hematocrit & $39 \%$ \\
\hline Mean corpuscular volume (MCV) & 85 \\
\hline Mean corpuscular hemoglobin concentration (MCHC) & $33 \% \mathrm{Hb} /$ cell \\
\hline Platelets & $215,000 / \mathrm{mm}^{3}$ \\
\hline WBC count & $8,500 / \mathrm{mm}^{3}$ \\
\hline Neutrophils & $59 \%$ \\
\hline Eosinophils & $1 \%$ \\
\hline Basophils & $0 \%$ \\
\hline Lymphocytes & $38 \%$ \\
\hline \multicolumn{2}{|l|}{ Urinanalysis } \\
\hline Glucose & Negative \\
\hline Bilirubin & Negative \\
\hline Ketone & Negative \\
\hline Specific gravity & 1.017 \\
\hline Blood & Negative \\
\hline $\mathrm{pH}$ & 6.3 \\
\hline Protein & Negative \\
\hline Nitrite & Negative \\
\hline Leukocytes & Negative \\
\hline \multicolumn{2}{|l|}{ Pulmonary function tests } \\
\hline $\mathrm{FEV}_{1} / \mathrm{FVC}$ & $72 \%$ \\
\hline \multicolumn{2}{|l|}{ Immunoglobulin test } \\
\hline Immunoglobulin $\mathrm{G}$ & $1,842 \mathrm{mg} / \mathrm{dL}$ \\
\hline IgG antibody, serum & Positive \\
\hline \multicolumn{2}{|l|}{ Bronchoalveolar lavage } \\
\hline \% lymphocytes & $>20 \%$ \\
\hline Mast cells & $1.8 \%$ \\
\hline
\end{tabular}

consistent with a diagnosis of BFL.

\section{Discussion}

BFL is most commonly reported after exposure to Psittaciformes order of birds such as parakeets, cockatoos and cockatiels as well as pigeons or doves. HP is usually encountered in the fourth to sixth decade of life with a mean age of 53 years [1]. The estimated incidence of interstitial lung disease is 7.6 cases per 100,000 persons per year of which HP accounted for $6.6 \%$ of those cases [1]. The overall prevalence of BFL among bird fanciers varies from $1.6 \%$ to $21 \%$ and smoking is related to lower prevalence but worse prognosis [1].
At each stage: acute, subacute or chronic, there are various diseases that present similarly to HP. In an acute setting of HP, the differentials include organic dust toxic syndrome (especially in farmers) or asthma. Chest radiographs in these cases are normal and respiratory symptoms include bronchial hyperreactivity and wheezing [2]. Subacute HP progresses to a more febrile disorder, with the differentials being infectious pneumonia or sarcoidosis. The key difference between sarcoidosis and HP however, is the presence of distinct granulomas in the lungs that follow a lymphangitic arrangement in sarcoidosis [2]. Idiopathic pulmonary fibrosis presents similarly to chronic HP, however the HRCT findings in idiopathic pulmonary fibrosis are predominantly in the basal segments of the lungs and classically present with a honeycomb appearance [2]. 
BAL of chronic HP shows an increased total cell count with a distinct rise in the $\mathrm{T}$ lymphocytes percentage (mostly greater than $50 \%$ ), these changes are not commonly seen in idiopathic pulmonary fibrosis. Chronic HP can be differentiated from interstitial pneumonia based on the histopathological findings showing fibrotic patches, fibroblast foci and subpleural honeycombing in interstitial pneumonia where else chronic HP presents with peribronchial fibrosis, bronchiolocentric inflammation, granulomas or multinucleated giants cells that may have cholesterol clefts, and bronchiolar epithelial hyperplasia on histopathological findings [2].

The diagnosis of BFL is based on a myriad of tests and clinical symptoms. There are six major criteria that are used for diagnosis [3]: 1) presence of clinical symptoms such as dyspnea, productive cough and fatigue, 2) confirmation of exposure to an agent, 3) consistent chest X-ray showing ground glass opacities, interstitial infiltrates and micronodules, 4) increased lymphocytes on BAL, 5) consistent histologic features which displays specific IgG antibodies against avian antigens and more well-formed, non-caseating granulomas in the interstitium, and 6) positive bronchial challenge test with PFTs demonstrating either an obstructive, restrictive or mixed pattern.

One of the most important features of BFL is the waxing and waning of symptoms when introduced to the inciting agent. Some of the physical finding include tachypnea and diffuse crackles. A reduction in diffusing capacity (DLCO) and mild arterial hypoxemia are also usually present. The presence of digital clubbing or an evidence of pulmonary fibrosis on surgical lung biopsy predicts a poorer prognosis.

Identification of the antigen responsible and removing exposure is of major importance in managing and preventing sensitization of the individual. This may be accomplished by minimizing contact with potential inciting agents, reducing microbial contamination of the work or home environment, or using protective equipment [4]. High levels of bird antigen can be detected in the setting for a continued period, up to 18 months, thus removal does not assure complete recovery. Glucocorticoids are the initial treatment for patients with subacute or chronic disease [4]. Therapy is usually initiated with prednisone, 0.5 to $1 \mathrm{mg}$ per kilogram of ideal body weight per day (up to a maximum daily dose of $60 \mathrm{mg}$ per day), given as a single dose each morning [4]. This dose is maintained for 1 to 2 weeks and then tapered over the next 2 to 4 weeks [4]. Lung transplantation can be a potential option for patients with chronic and progressive HP that is unresponsive to medical therapy [4].

\section{Consent}

Written informed consent was taken from the patient for publication of this case report and the accompanying images.

\section{References}

1. Hsieh C. (2016, September 09). Hypersensitivity pneumonitis. Retrieved September 05, 2017, from http:// emedicine.medscape.com/article/299174-overview.

2. Selman M, Pardo A, King TE, Jr. Hypersensitivity pneumonitis: insights in diagnosis and pathobiology. Am J Respir Crit Care Med. 2012;186(4):314-324.

3. Morell F, Roger A, Reyes L, Cruz MJ, Murio C, Munoz $\mathrm{X}$. Bird fancier's lung: a series of 86 patients. Medicine (Baltimore). 2008;87(2):110-130.

4. King TE, Jr. (2016, September 07). Treatment, prevention, and prognosis of hypersensitivity pneumonitis (extrinsic allergic alveolitis). Retrieved September 05, 2017, from https://www.uptodate.com/contents/treatment-prevention-and-prognosis-of-hypersensitivity-pneumonitisextrinsic-allergic-alveolitis. 